ZERTIFIZIERTE FORTBILDUNG

IN ZUSAMMENARBEIT MIT DER BAYERISCHEN LANDESÄRZTEKAMMER

Folge 145

Hier können Sie CME-Punkte sammeln a) für die Pflichtfortbildung aller Vertragsärzte und

b) für freiwillige Fortbildungszertifikate, die viele Landesärztekammern anbieten.

Die Multiple-Choice-Fragen beziehen sich auf den vorangegangenen Fortbildungsbeitrag (Seiten 40-43). Die Antworten ergeben sich z. T. aus dem Text, z. T. beruhen sie auf medizinischem Basiswissen!

Wenn Sie 70\% der Fragen richtig beantworten, erhalten Sie 2, bei 100\% 3 CME-Punkte.

Einsendeschluss: 22. März 2008 (www.cme-punkt.de) bzw. 6. Dezember 2007 (Teilnahme per Brief).
MMW

Fortschritte der Medizin

CME-Herausgeber- und Review-Board: Dr. H. J. K. Barwitz, Prof. Dr. A. Berghaus, Prof. Dr. Dr. h.c. Th. Brandt, Prof. Dr. W. G. Daniel, Prof. Dr W. Eisenmenger, Prof. Dr. K. Friese, Prof. Dr. H. S. Füessl, Prof. Dr. B. Göke, Prof. Dr. R. Gradinger Prof. Dr. Dr. h.c. A. Hofstetter, Prof. Dr. H. Holzgreve, Prof. Dr. A. Imdahl, Prof. Dr. K. Krüger, Prof. Dr. H.-J. Möller, Prof. Dr. Dr. h.c. G. Plewig, Prof. Dr. D. Reinhardt, U. Weigeldt.

\title{
Fragen zum Thema „COPD-Exazerbation“
}

- 1. Welche der folgenden Aussagen trifft zu?

A Die COPD geht mit einer reversiblen Atemwegsobstruktion einher.

B Der häufigste Grund für die Entstehung einer COPD sind Allergien.

C Die Lungenfunktion bleibt bei COPD auf einem einmal erreichten niedrigeren Niveau stehen.

D Anamnese, klinischer Befund und Lungenfunktionsprüfung sichern die Diagnose einer COPD.

E Klinische Mischbilder zwischen Asthma und COPD kommen nicht vor.

\section{- 2. Welche Maßnahme ist zur Prophylaxe und Therapie der COPD am erfolgreichs- ten?}

A Inhalative Kortikosteroide.

B Retardiertes Theophyllin.

C Anticholinergika 5\%.

D Nikotinkarenz.

E Rasch wirksame Beta-2-Mimetika.

\section{- 3. Was spricht am ehesten für eine bakte-} rielle Infektion der oberen Luftwege bei COPD?

A Leukozytose

B Fieber

C Reichlich gelbes Sputum

D Allgemeines Krankheitsgefühl

E Zunahme des Hustens

\section{- 4. Welche Aussage bezüglich der antibio-} tischen Therapie trifft nicht zu?

A Ein Antibiogramm ist für die Indikationsstellung für eine Antibiotikatherapie nicht erforderlich.
B Aminoglykoside sind Mittel der ersten Wahl bei akuten Exazerbationen einer COPD.

C Eine Röntgenaufnahme des Thorax ist für die Indikationsstellung zur antibiotischen Therapie nicht erforderlich.

D Begleitkrankheiten spielen für die Indikationsstellung zur antibiotischen Therapie eine wichtige Rolle.

E Die übliche Therapiedauer einer antibiotischen Therapie bei akut exazerbierter COPD beträgt sieben bis zehn Tage.

\section{Welches Kriterium spielt für die statio- näre Einweisung eines Patienten mit akut exazerbierter COPD die geringste Rolle? \\ A Dyspnoe und Zyanose \\ B Wesentliche Komorbiditäten \\ C Hohes Lebensalter \\ D Schlechte Blutgase bzw. Sauerstoff- sättigung \\ E Dauer der Erkrankung}

6. Welcher Erreger ist bei akut exazer-
bierter COPD wahrscheinlich nicht im
Spiel?
A Moraxella catarrhalis
B Staphylococcus epidermidis
C Streptococcus pneumoniae
D Haemophilus influenzae
E Pseudomonas aeruginosa

- 7. Welches ist der am besten evaluierte Parameter für die Bestimmung des Schweregrads einer Atemwegsobstruktion?

\begin{abstract}
A Peak-Flow-Messung
B Bodyplethysmografie

C Ein-Sekunden-Kapazität $\left(\mathrm{FEV}_{1}\right)$

D Röntgen-Thorax

E Lungenszintigrafie
\end{abstract}

\section{Welche der folgenden Aussagen trifft zu?}

A Die Pneumokokkenschutzimpfung ist alle zwei Jahre zu wiederholen.

B Eine Impfung gegen Pneumokokken ist bei COPD-Patienten nicht empfehlenswert.

C Eine Grippeschutzimpfung ist bei COPD-Patienten sehr riskant.

D Die Grippeschutzimpfung führt bei COPD-Patienten zu einer Reduktion von Pneumonien.

E COPD-Patienten unter inhalativen Steroiden dürfen wegen der stattfindenden Immunsuppression nicht geimpft werden.

\section{- 9. Welcher Auskultationsbefund spricht für eine bronchiale Obstruktion?}

A Leises Rauschen B Knistern

C Giemen D Rasseln

E Reiben

\section{Welches Antibiotikum spielt bei der kalkulierten Therapie der exazerbierten COPD die geringste Rolle?}

A Amoxicillin

B Metronidazol

C Ofloxacin

D Cefpodoxim

E Tetracyclin

\section{ANTWORTFORMULAR}

Mit Einreichen dieses Fragebogens erkläre ich mich damit einverstanden, dass die angegebenen Daten zum Zweck der Teilnahmebestätigung gespeichert und bei erfolgreicher Teilnahme an den Einheitlichen Informationsverteiler (EIV) der Ärztekammern weitergegeben werden.

Wichtiger Hinweis:

Eine Auswertung ist künftig nur noch möglich, wenn Sie Ihre EFN auf dem Antwortformular angeben! Nähere Hinweise hierzu unter: www.cme-punkt.de/faq.html
„COPD-Exazerbation"

MMW-Fortschr. Med. Nr. 47 / 2007 (149. Jg.)

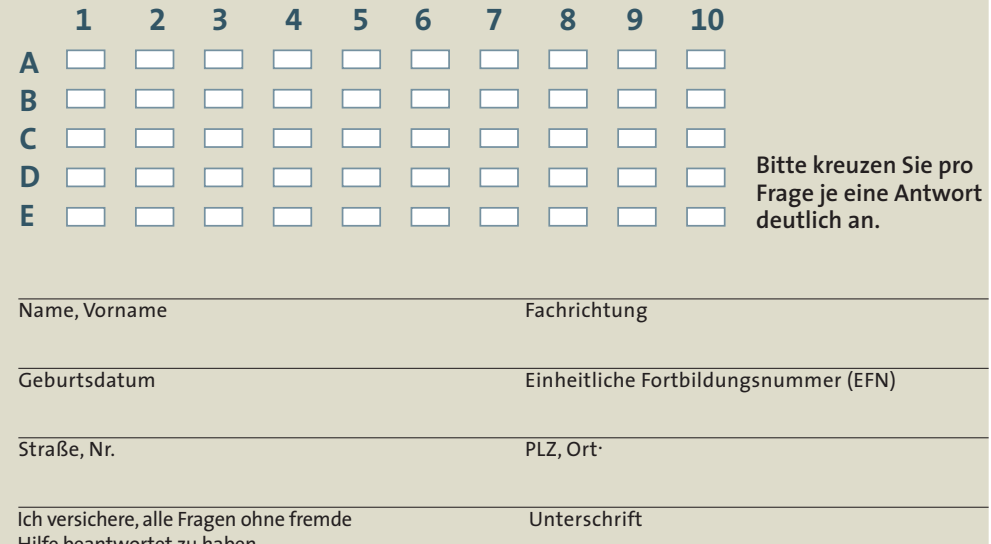

Und so kommen Sie zu Ihren Punkten

Teilnahme im Internet: unter www.cme-punkt.de. Dort führen wir für Sie ein elektronisches Punktekonto.

Teilnahme per Brief: Fragebogen ausfüllen und mit frankiertem Rückumschlag an Urban \& Vogel GmbH CME MMW-Fortschr. Med. Postfach 81664 München 